BIO Web of Conferences 1, 00081 (2011)

DOI: $10.1051 /$ bioconf $/ 20110100081$

(C) Owned by the authors, published by EDP Sciences, 2011

\title{
Constraints on Spontaneous Entrainment
}

\author{
R. C. Schmidt ${ }^{*} \quad$ Colleen Bucci ${ }^{* \dagger} \quad$ Michael J. Richardson ${ }^{\ddagger}$ \\ (*)College of the Holy Cross, USA \\ (*) College of the Holy Cross, USA \\ (†) University of Cincinnati, USA \\ E-mail: rschmidt@holycross.edu,cmbucc12@g.holycross.edu,richamo@ucmail.uc.edu
}

\begin{abstract}
Past research has revealed that a person's rhythmic limb movements become spontaneously entrained to an environmental rhythm if a.) visual information about the environmental rhythm is available and b.) its frequency of the environmental rhythm is near that of the person's movements. Further, this research has demonstrated that if the eyes track the environmental stimulus, the spontaneous entrainment to the environmental rhythm is strengthened. Experiments were performed to investigate two hypotheses that could explain this eye-tracking enhancement of spontaneous entrainment. One hypothesis is that eye tracking allows for the pick up of important coordinative information at the turn-around points of a movement trajectory. Another hypothesis is that the limb movements entrain to the moving eyes through a neuromotor synergy linking the eyes and limb. Results of these experiments will help delineate the informational and dynamical constraints that can impact the acquisition of skilled actions.
\end{abstract}

\section{Introduction}

Many common actions in one's daily life, such as dancing, jumping rope, or even stepping onto an escalator, require the synchronization of movements to a rhythmic event. A number of studies have investigated whether one's ability to synchronize to rhythmic environmental sources are constrained by dynamical processes of entrainment [1, 2, 3]. Research has shown two stable patterns of coordination, inphase $\left(\phi=0^{\circ}\right)$ and antiphase $\left(\phi=180^{\circ}\right)$, and that inphase coordination with a rhythmic stimulus is less variable than antiphase coordination [1]. These studies generally have found that coordination of the actor's movements and the environmental rhythm can be modeled by a coupled oscillator regime sustained by the perceptual medium of visual information.

Other research has revealed these dynamical processes of entrainment operate spontaneously to cause unintended entrainment of rhythmic limb movements to an environmental rhythm if a.) visual information about the environmental rhythm is available and b.) its frequency of the environmental rhythm is near that of the person's movements [4]. Interestingly this research has found that when subjects track a rhythmic stimulus with their eyes (as opposed to keeping them still), they are more likely to spontaneously entrain to the stimulus. One explanation of this eye-tracking enhancement of spontaneous entrainment is that tracking allows information about the endpoints contain of important direction change information which enhances rhythmic entrainment [5]. A second explanation of eye-tracking enhancement is that the movement of the eyes entrain the movements of the wrists due to a neuromuscular synergy: If the wrist is following the eyes and the eyes are following the stimulus, then the wrist would become entrained with the stimulus. In the current report, experiments investigating both these hypotheses will be described.

\section{Experiment 1: How important is direction change information for spontaneous entrainment?}

This experiment investigates whether adding trajectory direction change information to non-tracking conditions enhances spontaneous entrainment. Thirty undergraduate students from the College of the Holy Cross participated in the study in exchange for partial course credit. They were told that the study was investigating multitask performance and the effect of a distraction (i.e., pendulum swinging and oscillating stimulus on the screen) on a reaction time task (i.e., the reading the letters). What was really being studied was the unintentional entrainment of the participant's wrist movements to the stimulus oscillating and the visual information that was the basis for that entrainment. The participants swung the pendulum and verbally identified letters in either a.) an eye-tracking condition (the letters appeared on a square as it oscillated back and forth) or b.) in non-tracking conditions (the letters appeared on a stationary square which appeared under the trajectory of the oscillating stimulus). The placement of the stationary square was manipulated for

This is an Open Access article distributed under the terms of the Creative Commons Attribution-Noncommercial License 3.0, which permits unrestricted use, distribution, and reproduction in any noncommercial medium, provided the original work is properly cited. 
the non-tracking conditions so that direction change information was either available (under the left or right ends of the trajectory of the oscillating stimulus) or not (under the middle trajectory of the oscillating stimulus). A control condition in which letters appeared on a stationary square in the middle of the screen while an invisible oscillating stimulus oscillated back and forth was included to evaluate chance level entrainment. In addition, the oscillating square moved at three different frequencies, the subject's comfort mode period, this period $+5 \%$ (slower), and this period $-5 \%$ (faster). Trials were $40 \mathrm{~s}$ in length.

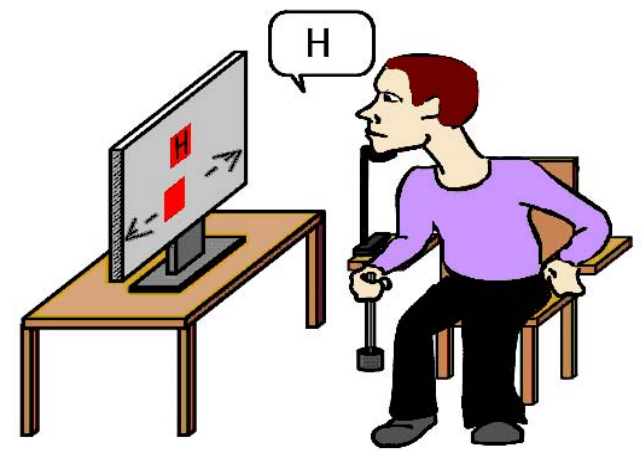

Figure 1. The experimental setting.

Thirty participants sat on a chair $60 \mathrm{~cm}$ from a 40 inch high-definition television screen (Sony KDL40XBR4), placed perpendicular to the screen (Figure 1). They were asked to place their chin in a chinrest positioned in front of the screen, and to swing a handheld pendulum in a sagittal plane while verbally identifying letters that appeared on the screen in the tracking, non-tracking or control conditions described above. The letters appeared randomly at time intervals between 0 and 2 seconds either on an oscillating or on a stationary square. Participants' wrist movements were measured at a sample rate of $100 \mathrm{~Hz}$ using an electrogoniometer attached to the back of the hand and $12-16 \mathrm{~cm}$ up the forearm. A hand-held pendulum was constructed from a wooden dowel $50 \mathrm{~cm}$ long with a $200 \mathrm{~g}$ plastic weight attached to its base. The pendulum had a natural period of $1.2 \mathrm{~s}$. The frequency of the oscillating visual stimulus was set to equal to .95 , 1 , or 1.05 times the subject's self-selected comfort mode period which was measured in the first trials of the experiment.

A $3 \times 3 \times 9$ repeated-measures ANOVA with variables of tracking (control, tracking and center nontracking) and stimulus frequency (slower, comfort, faster) and phase region $\left(0-20,20-40, \ldots, 160-180^{\circ}\right)$ was performed on the continuous relative phase angle that occurred between the participant's wrist movements and the environmental stimulus. The analysis replicated past results demonstrating that spontaneous inphase and antiphase entrainment for both the tracking and non-tracking conditions was greater than chance (Tracking $\mathrm{x}$ Region: $F(16,144)=$ 4.53, $p<.001, \eta_{p}{ }^{2}=0.34$ ) with a trend for entrainment being greater for tracking compared to non-tracking near antiphase. A mixed $3 \times 3 \times 9$ ANOVA with a between-subject variable of non-tracking placement (center, left and right) and within-subject variables of stimulus frequency (slower, comfort, faster) and phase region $\left(0-20,20-40, \ldots, 160-180^{\circ}\right)$ performed on the continuous relative phase revealed a significant interaction of non-tracking placement and phase region $\left(F(16,216)=2.63, p<.001, \eta_{p}^{2}=0.16\right)$ with the center placement condition exhibiting greater inphase entrainment than right and left (Figure 2). This result is counter to expectations that direction change information available at the left or right ends of trajectory would strengthen spontaneous entrainment and argues against the claim that eye tracking of a stimulus promotes greater entrainment because it allows direction change information to be picked up.

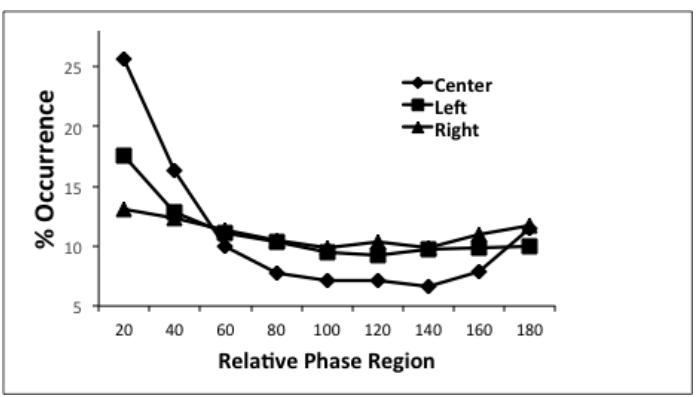

Figure 2. Comparison of spontaneous entrainment for nontracking conditions.

\section{Experiment 2: Will non-tracking rhythmic eye movements enhance entrainment?}

A second explanation for the eye-tracking enhancement of spontaneous entrainment is that the movement of the wrists begins to entrain the movements of the eyes, due to motor interference: If the wrist is following the eyes and the eyes are following the stimulus, then the wrist would become entrained with the stimulus. Such interference may be occur more strongly when the two movements are similar in in frequency and sinusoidal form. There is substantial body of past research that has found evidence for such a neuromotor synergy $[6,7]$. The current experiment investigates this hypothesis by asking whether any rhythmic eye movements facilitate entrainment. The task used changed the rhythmic 
movments of the eyes making them saccadic rhythmic eye movements - instead of smooth pursuit movements - in both horizontal and vertical planes cause spontaneous entrainment. If eye tracking saccadic eye movements in the horizontal plane reveals that limb movements become strongly spontaneously entrained, then of interest is whether the same results will occur when the eyes move in an even more different fashion, namely, in the vertical plane orthogonal to a horizontally moving stimulus.

Twenty-nine undergraduate students from the College of the Holy Cross participated in this study in exchange for partial course credit. The experimental task was identical to the previous experiment. Participants swung the pendulum and verbally identified letters under three conditions, a control condition, a non-tracking condition and a tracking condition. However, in the tracking or eye movement condition, the letters now appeared discretely on two squares placed horizontally on the screen at the rhythm of the oscillating stimulus that oscillated back and forth horizontally. Hence to read the letters, the participants needed to make not pursuit tracking movements but rather horizontal saccadic movements at the rhythm of the oscillating stimulus. Again of interest was the unintentional entrainment of the participant's wrist movements to the oscillating stimulus now that the eye movements have a kinematic form different from the sinusoidal wrist movements. In addition to moving their eyes horizontally, participants also performed trials in which they moved their eyes vertically. In these conditions, letters appeared on two squares placed vertically on the screen and letters discretely appeared on each square at the rhythm stimulus square that oscillated back and forth horizontally. Reading such letters required participants to move their eyes saccadically in the vertical plane.

In order to make the horizontal and vertical eye movements be rhythmic but have the reading of letters not be rhythmic, both letters and asterisks appeared in stationary boxes at the rhythm of the oscillating. Participants were told to ignore the asterisks and focus on reading the letters (which occurred randomly at the rate of the previous experiment every 1 to 3 seconds as in the previous experiment) as accurately and quickly as possible. This rhythmic display of letters and asterisks was used for the control and non-tracking conditions as well. Other than the rhythmic display of letters and asterisks, the non-tracking and control conditions were identical to the previous experiment. Also similar to the previous experiment, the oscillating square moved at three different frequencies, the subject's comfort mode period, this period $+5 \%$ (slower), and this period $-5 \%$ (faster).
For the horizontal eye movement task, a $3 \times 3 \times 9$ repeated-measures ANOVA with variables of tracking condition (control, saccadic tracking and non-tracking) and stimulus frequency (slower, comfort, faster) and phase region $\left(0-20,20-40, \ldots, 160-180^{\circ}\right)$ was performed on the continuous relative phase angle that occurred between the participant's wrist movements and the environmental stimulus. The analysis revealed a significant interaction of tracking condition and phase region, $F(16,208)=4.116, \mathrm{p}=.001, \eta_{\mathrm{p}}{ }^{2}=.240$, indicating that spontaneous inphase entrainment occurred for horizontal saccadic eye movements (Figure 3, top).

For the vertical eye movement task, an identical $3 \mathrm{x}$ 3 X 9 repeated-measures ANOVA was performed on the continuous relative phase angle. This analysis revealed nonsignificant effects indicating that spontaneous inphase entrainment did not occur when the eyes moved rhythmically in the vertical plane tracking (Figure 3, bottom).
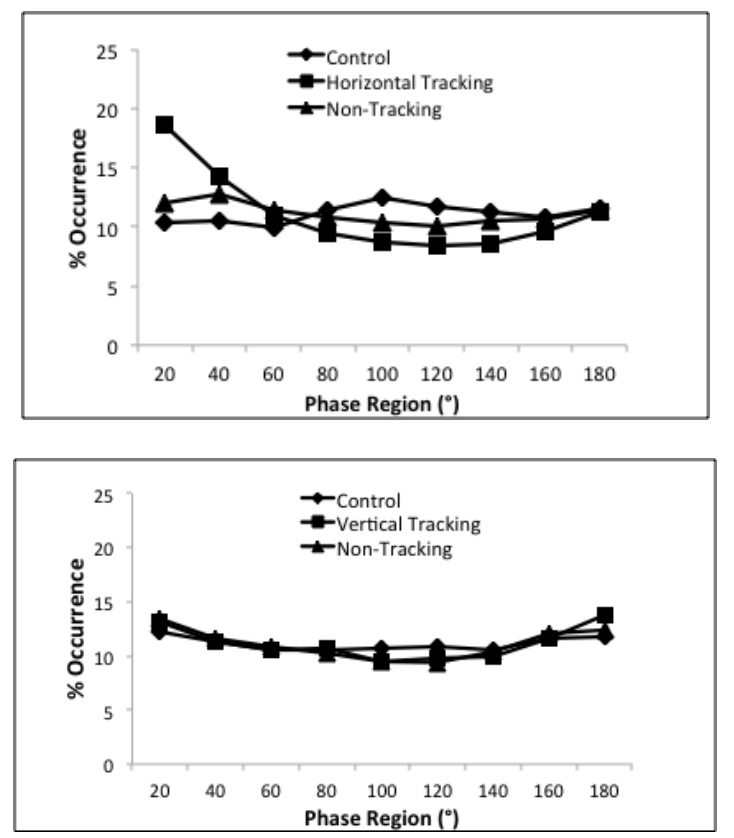

Figure 3. Spontaneous entrainment was found for horizontal (top) but not vertical (bottom) saccadic eye movement conditions.

\section{Conclusions}

The aim of these experiments was to understand the constraints on eye movement-enhanced spontaneous entrainment to a rhythmic stimulus. There are two general explanations of this phenomenon. The first is that eye movements enhance the perceptual coupling of the performer's movements to the rhythmic stimulus by increasing the amount or quality of the perceptual 
information available. The second explanation is that a neuromotor synergy between the eye movements and wrist movements plays an intermediary role in entraining the wrist movements to the rhythmic stimulus.

Experiment 1 provides conclusive evidence that the eye movement-enhanced spontaneous entrainment is not a consequence of the perceptual coupling being enhanced by increased direction change information: Left and right side non-tracking conditions (which contain direction change information) failed to enhance spontaneous entrainment over the center non-tracking condition (which did not contain direction change information). It still remains possible however that eye movements strengthen the perceptual coupling by virtue of allowing more information about the stimulus' movement to be picked up. The relative strength of center tracking over right and left tracking supports this possibility (see Figure 2).

Experiment 2 investigated the role of a neuromotor synergy in eye movement-enhanced spontaneous entrainment by changing the kinematic characteristics of the eye movements being executed. Having the eyes move in a saccadic rather than pursuit fashion did not eliminate spontaneous entrainment of the wrist to the rhythmic stimulus but having the eyes move vertically rather than horizontally did. These results suggest that not any kind of rhythmic eye movements even if they are at the same tempo of the stimulus facilitates spontaneous entrainment. Of course, the horizontal saccadic movement that did increase entrainment still allow for the pick up of additional information about the stimulus' kinematics that may enhance the perceptual coupling between the wrist and the rhythmic stimulus. An entrainment experiment currently underway that uses a rhythmic stimulus moving in depth in which full kinematic information is available but the eyes never move will allow us to more fully evaluate the role of perceptual or neuromotor processes in eye movement-enhanced spontaneous entrainment.

The investigation of the spontaneous entrainment to environmental rhythms while not skilled performance as such explores the implicit context of constraint for the acquisition of skilled actions. Understanding the lawful dynamical processes that form the selforganizing milieu for interacting with the environment as well as the information constraints that condition such self-organization are important for determining what a skilled performer can implicitly rely upon in acquiring motor expertise.

\section{References}

[1] Kelso, J. A. S., DelColle, J. D., \& Schoner, G. (1990). Action-perception as a pattern formation process. In M.
Jeannerod (Ed.), Attention and performance XIII (Vol. 5, pp. 139-169). Hillsdale, NJ: Erlbaum.

[2] Wimmers, R. H., Beek, P. J., \& Van Wieringen, P. C. (1992). Phase transitions in rhythmic tracking movements: A case of unilateral coupling. Human Movement Science, 11, 217-226.

[3] Russell, D. M., \& Sternad, D. (2001). Sinusoidal visuomotor tracking: Intermittent servo-control or coupled oscillations? Journal of Motor Behavior, 33(4), 329-349.

[4] Schmidt, R. C., Richardson, M. J., Arsenault, C. A., \& Galantucci, B. (2007). Visual tracking and entrainment to an environmental rhythm. Journal of Experimental Psychology: Human Perception and Performance, 33, 860-870.

[5] Hajnal, A., Richardson, M. J., Harrison, S. J., \& Schmidt, R. C. (2009). Location but not amount of stimulus occlusion influences the stability of visuomotor coordination. Experimental Brain Research, 199, 89-93.

[6] van Donkelaar, P., Siu, K.-C., \& Walterschied, J. (2004). Saccadic output is influenced by limb kinetics during eye-hand coordination. Journal of Motor Behavior, 36, 245-252.

[7] Henriques, D. Y. P., \& Crawford, J. D. (2002). Role of eye, head, and shoulder geometry in the planning of accurate arm movements. Journal of Neurophysiology, $87,1677-1685$ 